\title{
Research on the influence of focusing function based on different texture image
}

\author{
Ren shuyan ${ }^{1, a^{*}}$, Lu liu ${ }^{2, b}$ \\ ${ }^{1}$ Key laboratory of Information Sensing\&Intelligent Control, Tianjin University of Technology and \\ Education, Tianjin 300222, China \\ ashuyan_ren@163.com, b1421640150@qq.com
}

\begin{abstract}
Keywords: texture image, clarity evaluation function, focus
Abstract. The main research content of this paper is to take fabric images with different texture features, by three kinds of commonly used and relatively good performance of the clarity evaluation function process the image, such as gray scale gradient function, energy gradient function (Tenengrad function) and Laplasse algorithm, So as to obtain the clarity evaluation function curve of different texture images, and then according to the characteristics of the single peak, no bias and anti interference ability, draw texture image that is much more detail-rich images easier to focus.
\end{abstract}

\section{Introduction}

With the development of science and technology, automatic focusing is one of the key technologies in the system imaging, which has been widely used in many fields, such as digital camera, video surveillance, medical imaging, computer vision and so on. In the textile production, quality control is very important, fabric defect detection is one of the most important parts, before the camera fabric image, with the camera focusing to can get clear and less interference with different texture of the fabric image, to facilitate subsequent processing of the image. The process of automatic focusing of image is: First, using imaging equipment for different focus on the image of the shooting sample, the resulting image sequence is converted into a computer after a digital conversion. Then, the computer calculates the specific region of the image sequence by focusing evaluation function, to evaluate the degree of focusing. Finally, the pole searching algorithm to obtain focused images, that is the biggest value of the image evaluation function, Shoot and save the image to the imaging device. The focus of the system mainly depends on image sharpness, therefore, it is the key technology of automatic focusing to judge the clarity of the image. Objectively, the details of the image and the edge information determine the clarity of the image. From the basic principle of auto focus, compared with defocused image, the details of the focused image are more abundant and contain more information, on the time-domain representation of edges are sharp, the frequency domain representation of high frequency components is more. At present, the evaluation function of the definition is divided into two types of the frequency domain and the spatial domain, the frequency domain function needs to be transformed from the airspace to the frequency domain, the computation quantity is large and complex, but the calculation of spatial evaluation function is small and simple. Through the gray gradient function, energy gradient function (Tenengrad function) and Laplace algorithm, this three definition evaluation function evaluate different fabric texture images from the defocus -the focus-the defocus, the result is the texture of the image, the more details, the characteristics of sharpness function is better, the image becomes easier to focus.

\section{Several commonly used definition evaluation function}

Image sharpness evaluation function plays a key role in the automatic focusing technology based on image processing, the basis for judgment: When the image is in focus, the image is the most clear, the image details are the most abundant, and has the biggest evaluation function value; With increasing degree of defocus, the image is gradually blurred, and the value of the evaluation function is also decreased. The evaluation function of the auto focus should have the characteristics of single peak, no 
bias, robustness, real time and so on, in the vicinity of the peak function value change rate is high. Commonly used definition evaluation function: gray gradient function, energy gradient function and Laplasse algorithm. The ideal evaluation function curve is shown in Fig. 1.

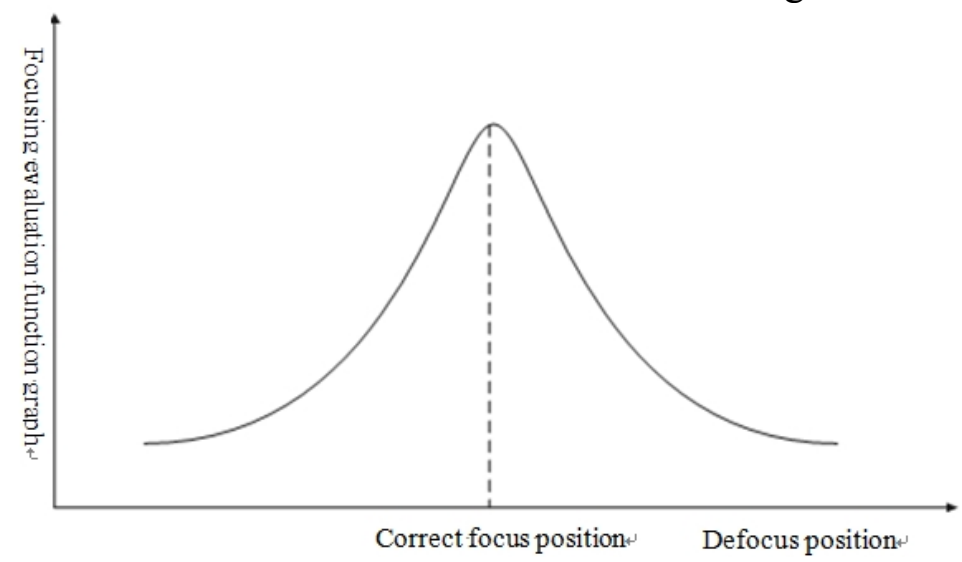

Fig. 1 the most ideal evaluation function curve

Gray gradient function. Gray gradient function is one of the most commonly used evaluation functions, and the application of gray gradient function is the premise that the image contains more sharp edge information, the greater the gradient value, the more information the image edge information. $\mathrm{S}(\mathrm{x}, \mathrm{y})$ as the evaluation function value, it is the absolute value of the gray value of two adjacent pixels in the image. When the function value $\mathrm{S}(\mathrm{x}, \mathrm{y})$ is the maximum, it is focus position, the evaluation function is:

$$
S(x, y)=\sum_{x, y}\{|f(x, y)-f(x, y-1)|+|f(x, y)-f(x-1, y)|\}
$$

When the $\mathrm{S}$ takes the maximum value, indicating that the state is already in a fully focused imageis the sharpest. However, sometimes because the difference in the gray value of the adjacent pixels is not too large, it is easy to cause the evaluation function curve is not obvious, or simply do not appear extreme points, so sometimes (1) type and can not reflect the quality of the image, so on the basis of this function, write an improved algorithm, such as (2):

$$
S(x, y)=\sum_{x, y}\left\{I_{x}^{2}(x, y)+I_{y}^{2}(x, y)\right\}
$$

Among them $I_{x}(x, y)=f(x+1, y)-f(x, y) \quad, \quad I_{y}(x, y)=f(x, y+1)-f(x, y) \quad . \quad$ Take $S_{0}=\underset{1 \leq k \leq N}{\operatorname{MAX}}\{S(x, y)\}$, At this time $\mathrm{S}_{0}$ for the best focus.

Energy gradient function (Tenengrad function). The energy gradient function of the image can be defined as shown in (3):

$$
S(x, y)=\sqrt{f_{x k}^{2}(x, y)+f_{y k}^{2}(x, y)}
$$

Among them $f_{x k}(x, y)=I_{x} * f(x, y), f_{y k}(x, y)=I_{y} * f(x, y) . f(x, y)$ for the gray value of the image, $I_{x}, I_{y}$ for the Sobel operator, * for the convolution,

$$
I_{x}=\left[\begin{array}{lll}
-1 & 0 & 1 \\
-2 & 0 & 2 \\
-1 & 0 & 1
\end{array}\right], I_{y}=\left[\begin{array}{ccc}
1 & 2 & 1 \\
0 & 0 & 0 \\
-1 & -2 & -1
\end{array}\right]
$$

Take $S_{0}=\underset{1 \leq k \leq N}{\operatorname{MAX}}\{S(x, y)\}$, At this time $\mathrm{S}_{0}$ for the best focus. 
Laplasse algorithm. By using Laplasse operator to calculate the image of the two order differential and the sum of the image of the two order differential as the basis for the evaluation of the image sharpness, the image of the two order differential operation:

$$
\nabla^{2} f(x, y)=\frac{\partial^{2} f(x, y)}{\partial x^{2}}+\frac{\partial^{2} f(x, y)}{\partial y^{2}}
$$

The two order differential corresponding to different differential methods have different templates, for the following template:

\begin{tabular}{|c|c|c|}
\hline 0 & 1 & 0 \\
\hline 1 & -4 & 1 \\
\hline 0 & 1 & 0 \\
\hline
\end{tabular}

The evaluation function is computed as follows:

$$
S(x, y)=\sum_{x, y}\left(g_{x}+g_{y}\right)^{2}
$$

Among them $g_{x}+g_{y}=I(i+1, j)+I(i-1, j)+I(i, j+1)+I(i, j-1)-4 I(i, j), I(x, y)$ was the gray value at point $(x, y)$, Take $S_{0}=\underset{1 \leq k \leq N}{\operatorname{MAX}}\{S(x, y)\}$, At this time $S_{0}$ for the best focus.

\section{Experimental analysis}

The sequence images of the different degree of the defocus image are obtained by focusing shooting, according to the method of the defocus -the focus-the defocus on the image, the image sharpness value is calculated. In this paper, the degree of difficulty in focusing on different texture features is studied Selected samples of two kinds of fabric, respectively for multi texture and less texture image, a comparison of face images with more texture features is shot at the same time, and draws the corresponding conclusion.
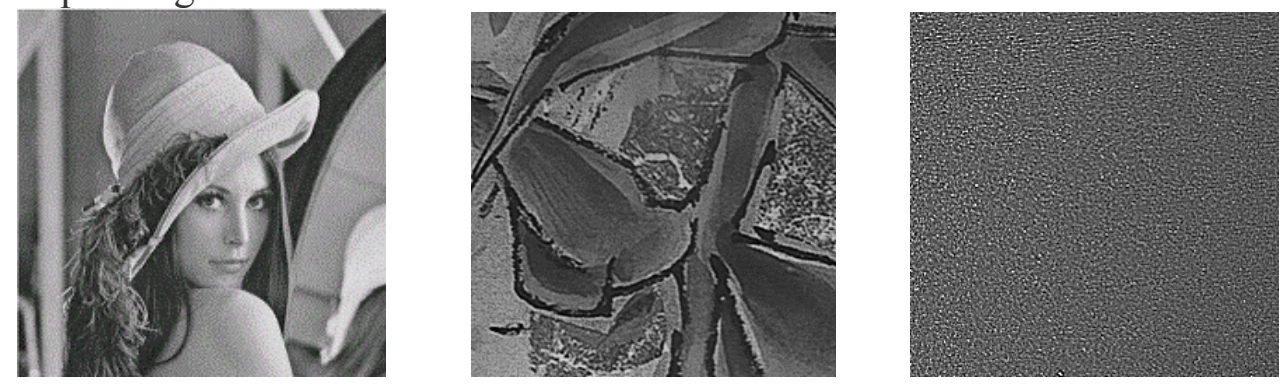

Fig. 2 the texture features gradually reduce from the left to the right image

This simulation experiment took three groups of images, each group has 20 different degrees of focal image, select 9 images in each group, the sequence of images is the defocus-the focus-the defocus, using these images, the curves of three kinds of evaluation functions are obtained. Abscissa is the serial number of the image, the vertical is the normalized evaluation function value. In Figure 3, figure (a) for image gray gradient function evaluation curve, figure (b) for the image energy gradient function evaluation curve, figure (c) for the image Laplacian evaluation curve. In Figure 3, the "+" represents the normalized value of the face image. "*" represents the multi texture fabric image normalized evaluation function value, "^" represents the less texture fabric image normalized evaluation function value. 


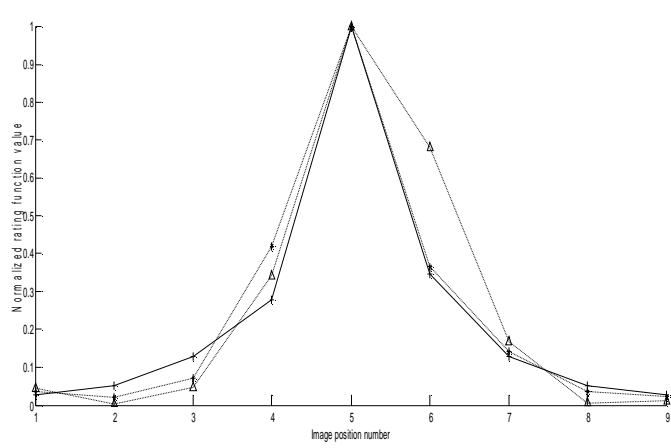

figure (a)

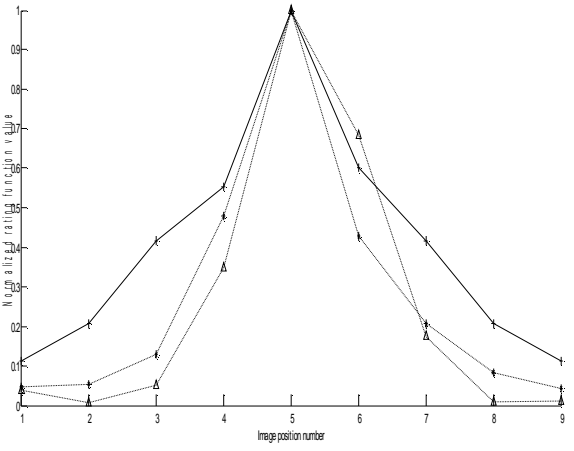

figure (b)

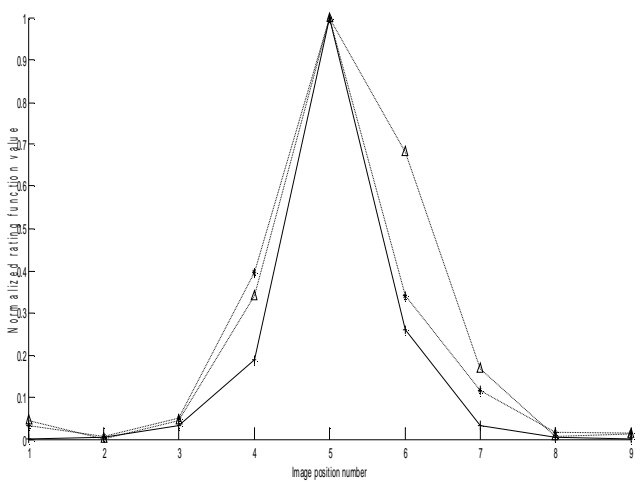

figure (c)

From the results of the above analysis, how much of the image texture is very big impact on the performance of the focus. The face image is rich in detail, and the evaluation function has the extreme value in the image focus. The evaluation function can satisfy the single peak and no bias, and can accurately position the focusing position; Less texture of fabric images lack of detail, the evaluation function curve will fluctuate, and function in the near the peak value change rate is not particularly obvious. As a result, the stability is low; Multi texture fabric image contains more detailed information, the evaluation function curve characteristics than the characteristics of face image difference but the characteristics of the fabric texture image well. From the above experimental results, the more texture features can be included, the more details is contained, the image is focused more easily.

\section{Summary}

In digital image processing, the definition of digital image is an important content, which is of great significance to realize the auto focusing of precision instruments. Experiments show that the more abundant texture features of the image more easy to achieve automatic focus. This is just a study of the static scene, the next step is to focus on the focus of the movement target.

\section{References}

[1] Gao zhan. Accuracy and stability of the auto focusing evaluation function [D]. Shandong University, 2007.

[2] Zheng yuanyuan. Auto focus algorithm research [D]. Shandong University, 2012.

[3] Wang haijuan.Wang guoyu.Ding wen. The study defocused image clarity evaluation function [J] Microcomputer Information, 2011,08: 166-167.

[4] Zhang guang. HD network camera auto-focus technology research [D]. Zhengzhou University, 2014. 